ANNALES

POLONICI MATHEMATICI

$88.2(2006)$

\title{
Explicit difference schemes for nonlinear differential functional parabolic equations with time dependent coefficients - convergence analysis
}

\author{
by A. Poliński (Gdańsk)
}

\begin{abstract}
We study the initial-value problem for parabolic equations with time dependent coefficients and with nonlinear and nonlocal right-hand sides. Nonlocal terms appear in the unknown function and its gradient. We analyze convergence of explicit finite difference schemes by means of discrete fundamental solutions.
\end{abstract}

1. Introduction. The paper presents a convergence analysis for explicit finite difference methods (FDM's) consistent with parabolic equations whose leading terms are time dependent. The right-hand side contains nontrivial nonlocal operators (delays, integrals), acting on the unknown function and its derivatives. We show that discrete solutions and their spatial difference quotients converge uniformly to the exact solution and its gradient. Unlike [1], [4], [7], [8] the maximum principle is not applicable in that case because the gradient essentially depends on functional arguments (delays, integrals). We generalize some results of [6], where an analogous convergence theorem has been proven for a nonlocal heat equation, i.e., the leading term was just the Laplacean. Our nonlocal terms, main assumptions and ideas are basically the same. We exploit the concept of matching sequences of indices, which leads to an a priori estimate of differences of the discrete fundamental solution. Unlike [6] we do not arrive at these estimates by direct analysis of paired sequences, but by an elegant combinatorial Lemma 4, whose idea is adapted from some results of Littlewood, Offord, Katona and Kleitman [5]. Owing to this lemma it becomes possible to estimate complicated expressions in the presence of variable coefficients $a_{k}(t)$.

The organization of the paper is as follows: 1) formulation of the differential-functional problem and standard assumptions on coercivity and bound-

2000 Mathematics Subject Classification: Primary 35R10; Secondary 65M06, 65M12, $35 \mathrm{~K} 15$.

Key words and phrases: finite difference, stability, parabolic, nonlocal. 
edness of the leading term, 2) formulation of the difference scheme and auxiliary lemmas on the positivity of its coefficients and properties of a discrete fundamental solution, 3) the crucial Lemma 5 on estimates of finite differences between two values of the fundamental solution, 4) formulation of assumptions on the right-hand side function, natural in the consistency and stability theory of difference schemes, 5) deduction of the convergence theorem from the consistency and stability lemmas, 6) numerical experiments in $\mathbb{R}^{2}$.

1.1. Formulation of the differential and difference problem. Given $d>0$ and $\tau_{0}, \tau_{1}, \ldots, \tau_{n} \in \mathbb{R}_{+}$, set $[-\tau, \tau]=\left[-\tau_{1}, \tau_{1}\right] \times \ldots \times\left[-\tau_{n}, \tau_{n}\right], E=[0, d]$ $\times \mathbb{R}^{n}, E_{0}=\left[-\tau_{0}, 0\right] \times \mathbb{R}^{n}$ and $B=\left[-\tau_{0}, 0\right] \times[-\tau, \tau]$. Denote by $C(B, \mathbb{R})$ the set of all continuous functions from $B$ to $\mathbb{R}$. If $u: E_{0} \cup E \rightarrow \mathbb{R}$ and $(t, x) \in E$, then we define the Hale-type functional $u_{(t, x)}: B \rightarrow \mathbb{R}$ by $u_{(t, x)}(s, y)=$ $u(t+s, x+y)$ for $(s, y) \in B$. If $U=\left(u_{1}, \ldots, u_{n}\right): E_{0} \cup E \rightarrow \mathbb{R}^{n}$, then $U_{(t, x)}=\left(\left(u_{1}\right)_{(t, x)}, \ldots,\left(u_{n}\right)_{(t, x)}\right)$.

Suppose that $a_{k}:[0, d] \rightarrow \mathbb{R}$ and $\phi: E_{0} \rightarrow \mathbb{R}$ are continuous, $f: \Omega \rightarrow \mathbb{R}$, where $\Omega:=E \times C(B, \mathbb{R}) \times C\left(B, \mathbb{R}^{n}\right)$. Consider the Cauchy problem

$$
\begin{gathered}
\partial_{t} u(t, x)=\sum_{k=1}^{n} a_{k}(t) \partial_{x_{k} x_{k}} u(t, x)+f\left(t, x, u_{(t, x)},\left(\partial_{x} u\right)_{(t, x)}\right) \quad \text { on } E, \\
u(t, x)=\phi(t, x) \quad \text { on } E_{0} .
\end{gathered}
$$

We will use the following assumptions; the first implies coercivity of the differential operator $-\sum_{k=1}^{n} a_{k}(t) \partial_{x_{k} x_{k}}$, whereas the second guarantees the stability of the difference problem, as well as the uniform boundedness of the coefficients $a_{k}(\cdot)$.

Assumption 1. $a_{k}(t) \geq \varepsilon_{0}>0$ for $k=1, \ldots, n$ and $t \in[0, d]$.

Assumption 2. There are positive numbers $C_{1}, \ldots, C_{n}$ such that

$$
1-\sum_{k=1}^{n} 2 C_{k}^{2} a_{k}(t) \geq 0, \quad t \in[0, d] .
$$

Fix $C_{1}, \ldots, C_{n} \in(0, \infty)$. Define the set of admissible steps:

$$
\begin{aligned}
I_{C}=\left\{h=\left(h_{0}, h^{\prime}\right): h_{0} \in(0, d), h^{\prime}=\left(h_{1}, \ldots, h_{n}\right) \in \mathbb{R}_{+}^{n},\right. \\
\\
\left.h_{i}^{2} C_{i}^{2}=h_{0}(i=1, \ldots, n)\right\} .
\end{aligned}
$$

It is seen that the time step $h_{0}$ is proportional to $h_{i}^{2}$. Recall that explicit schemes are stable for sufficiently small coefficients $C_{i}$.

We introduce a regular mesh. Set $t^{\alpha}=\alpha h_{0}$ and $x^{\beta}=\left(\beta_{1} h_{1}, \ldots, \beta_{n} h_{n}\right)$ for $\alpha \in \mathbb{Z}$ and $\beta \in \mathbb{Z}^{n}$. Let $Z_{h}=\left\{\left(t^{\alpha}, x^{\beta}\right):(\alpha, \beta) \in \mathbb{Z}^{1+n}\right\}$. Define $E_{h}^{0}=E_{0} \cap Z_{h}$, $E_{h}=E \cap Z_{h}, \widetilde{E}_{h}=E_{h}^{0} \cup E_{h}, E_{h}^{+}=\left\{\left(t^{\alpha}, x^{\beta}\right) \in E_{h}:\left(t^{\alpha+1}, x^{\beta}\right) \in E_{h}\right\}, B_{h}=$ 
$B \cap Z_{h}$. We will use the difference operators $\delta_{0}^{+}, \delta_{k}$ and $\delta_{k k}(k=1, \ldots, n)$ defined by

$$
\begin{aligned}
\delta_{0}^{+} u^{(\alpha, \beta)} & =\frac{u^{(\alpha+1, \beta)}-u^{(\alpha, \beta)}}{h_{0}}, \\
\delta_{k} u^{(\alpha, \beta)} & =\frac{u^{\left(\alpha, \beta+e_{k}\right)}-u^{\left(\alpha, \beta-e_{k}\right)}}{2 h_{k}}, \\
\delta_{k k} u^{(\alpha, \beta)} & =\frac{u^{\left(\alpha, \beta+e_{k}\right)}-2 u^{(\alpha, \beta)}+u^{\left(\alpha, \beta-e_{k}\right)}}{h_{k}^{2}},
\end{aligned}
$$

where $e_{k}=\left(\delta_{1, k}, \ldots, \delta_{n, k}\right)$ and $\delta_{j, k}$ is the Kronecker symbol for $j, k=$ $1, \ldots, n$. The difference operators $\delta_{0}^{+}, \delta_{k}, \delta_{k k}$ approximate the respective partial derivatives $\partial_{t}, \partial_{x_{k}}$ and $\partial_{x_{k} x_{k}}$. For all $t^{\alpha} \in[0, d]$ define

$$
c_{0}^{(\alpha)}=1-\sum_{k=1}^{n} \frac{2 h_{0}}{h_{k}^{2}} a_{k}\left(t^{\alpha}\right), \quad c_{ \pm e_{k}}^{(\alpha)}=\frac{h_{0}}{h_{k}^{2}} a_{k}\left(t^{\alpha}\right) \quad(k=1, \ldots, n) .
$$

Put $c_{s}^{(\alpha)}=0$ for all remaining multiindices $s \in \mathbb{Z}^{n}$ and all $\alpha \geq 0$.

LEMMA 1. If Assumptions 1 and 2 are satisfied and $h \in I_{C}$, then

$$
\sum_{s \in \mathbb{Z}^{n}} c_{s}^{(\alpha)}=1, \quad c_{s}^{(\alpha)} \geq 0, \quad \text { for } t^{\alpha} \in[0, d], s \in \mathbb{Z}^{n}
$$

The finite difference approximation of problem (1)-(2) takes the form

$$
\begin{gathered}
\delta_{0}^{+} u^{(\alpha, \beta)}=\sum_{k=1}^{n} a_{k}^{(\alpha)} \delta_{k k} u^{(\alpha, \beta)}+f[u]^{(\alpha, \beta)} \quad \text { on } E_{h}^{+} \\
u^{(\alpha, \beta)}=\bar{\phi}^{(\alpha, \beta)} \quad \text { on } E_{h}^{0}
\end{gathered}
$$

where $a_{k}^{(\alpha)}=a_{k}\left(t^{\alpha}\right), \bar{\phi}$ is a discrete perturbed counterpart of $\phi$,

$$
\begin{gathered}
f[u]^{(\alpha, \beta)}=f_{h}\left(t^{\alpha}, x^{\beta}, u_{[\alpha, \beta]},(\delta u)_{[\alpha, \beta]}\right), \quad \delta u=\left(\delta_{1} u, \ldots, \delta_{n} u\right), \\
u_{[\alpha, \beta]}\left(t^{\widetilde{\alpha}}, x^{\widetilde{\beta}}\right)=u\left(t^{\widetilde{\alpha}+\alpha}, x^{\widetilde{\beta}+\beta}\right) \quad \text { for }\left(t^{\widetilde{\alpha}}, x^{\widetilde{\beta}}\right) \in B_{h}, \\
(\delta u)_{[\alpha, \beta]}=\left(\left(\delta_{1} u\right)_{[\alpha, \beta]}, \ldots,\left(\delta_{n} u\right)_{[\alpha, \beta]}\right),
\end{gathered}
$$

and $f_{h}: E_{h}^{+} \times B_{h} \mathbb{R} \times\left({ }^{B_{h}} \mathbb{R}\right)^{n} \rightarrow \mathbb{R}$. Here ${ }^{B_{h}} \mathbb{R}$ denotes the set of all real functions defined on $B_{h}$. Equation (4) can be rewritten in the explicit form

$$
u^{(\alpha+1, \beta)}=\sum_{s \in \mathbb{Z}^{n}} c_{s}^{(\alpha)} u^{(\alpha, \beta+s)}+h_{0} f[u]^{(\alpha, \beta)} .
$$

Formula (7) is crucial in our further theoretical considerations. First we investigate basic properties of solutions of such equations. 


\subsection{Auxiliary statements}

LEMma 2. Suppose that Assumptions 1 and 2 are satisfied. If $h \in I_{C}$, $g: E_{h}^{+} \rightarrow \mathbb{R}$ and $u: \widetilde{E}_{h} \rightarrow \mathbb{R}$ satisfies the equation

$$
u^{(\alpha+1, \beta)}=\sum_{s \in \mathbb{Z}^{n}} c_{s}^{(\alpha)} u^{(\alpha, \beta+s)}+h_{0} g^{(\alpha, \beta)} \quad \text { on } E_{h}^{+},
$$

then

$$
\begin{aligned}
\|u\|_{\infty}(\alpha+1) & \leq\|u\|_{\infty}(0)+h_{0} \sum_{\mu=0}^{\alpha}\|g\|_{\infty}(\mu) \\
& \leq\|u\|_{\infty}(0)+t^{\alpha+1}\|g\|_{\infty}(\alpha),
\end{aligned}
$$

where $\|v\|_{\infty}(\alpha)=\sup _{\widetilde{\alpha} \leq \alpha, \beta \in \mathbb{Z}^{n}}\left|v^{(\widetilde{\alpha}, \beta)}\right|$ for any discrete function $(\alpha, \beta) \mapsto$ $v^{(\alpha, \beta)}$, and there exists a unique representation of the solution

$$
\begin{aligned}
u^{(\alpha, \beta)}= & \sum_{\eta \in \mathbb{Z}^{n}} \Gamma^{(\alpha, \beta, 0, \eta)} u^{(0, \eta)} \\
& +h_{0} \sum_{\zeta=1}^{\alpha} \sum_{\eta \in \mathbb{Z}^{n}} \Gamma^{(\alpha, \beta, \zeta, \eta)} g^{(\zeta-1, \eta)} \quad \text { on } E_{h},
\end{aligned}
$$

where $\Gamma^{(\alpha, \beta, \zeta, \eta)}$ is the discrete fundamental solution, determined by the recurrence relations

$$
\begin{aligned}
\Gamma^{(\alpha, \beta, \alpha, \eta)} & =\delta_{0,|\beta-\eta|}, \\
\Gamma^{(\alpha+1, \beta, \zeta, \eta)} & =\sum_{s \in \mathbb{Z}^{n}} c_{s}^{(\alpha)} \Gamma^{(\alpha, \beta+s, \zeta, \eta)}, \quad 0 \leq \zeta \leq \alpha,
\end{aligned}
$$

where $\delta_{0,|\beta-\eta|}$ is the Kronecker symbol.

Proof. It follows from (8) that

$$
\left|u^{(\alpha+1, \beta)}\right| \leq \sum_{s \in \mathbb{Z}^{n}}\left|c_{s}^{(\alpha)} u^{(\alpha, \beta+s)}\right|+h_{0}\left|g^{(\alpha, \beta)}\right| .
$$

By Lemma 1 we have the recurrence inequality

$$
\|u\|_{\infty}(\alpha+1) \leq\|u\|_{\infty}(\alpha)+h_{0}\|g\|_{\infty}(\alpha) .
$$

This completes the proof of estimate (9).

Now we prove (10)-(12) by induction on $\alpha$. For $\alpha=0,(10)$ follows from (11), which is obvious. Assume that (10) holds for some $\alpha \geq 0$. It follows from (8), (10) and (12) that

$$
\begin{aligned}
u^{(\alpha+1, \beta)}= & \sum_{s \in \mathbb{Z}^{n}} c_{s}^{(\alpha)}\left\{\sum_{\eta \in \mathbb{Z}^{n}} \Gamma^{(\alpha, \beta+s, 0, \eta)} u^{(0, \eta)}+h_{0} \sum_{\zeta=1}^{\alpha} \sum_{\eta \in \mathbb{Z}^{n}} \Gamma^{(\alpha, \beta+s, \zeta, \eta)} g^{(\zeta-1, \eta)}\right\} \\
& +h_{0} g^{(\alpha, \beta)}
\end{aligned}
$$




$$
\begin{aligned}
= & \sum_{\eta \in \mathbb{Z}^{n}} u^{(0, \eta)} \sum_{s \in \mathbb{Z}^{n}} c_{s}^{(\alpha)} \Gamma^{(\alpha, \beta+s, 0, \eta)} \\
& +h_{0} \sum_{\zeta=1}^{\alpha} \sum_{\eta \in \mathbb{Z}^{n}} g^{(\zeta-1, \eta)} \sum_{s \in \mathbb{Z}^{n}} c_{s}^{(\alpha)} \Gamma^{(\alpha, \beta+s, \zeta, \eta)}+h_{0} g^{(\alpha, \beta)}
\end{aligned}
$$

Since this relation holds for arbitrary functions $u^{(0, \eta)}$ and $g$, we have (12), and (10) for $\alpha+1$. This completes the proof.

REMARK 1. It follows from Lemma 2 that

$$
\Gamma^{(\alpha, \beta, \zeta, \eta)}=\sum_{s_{1} \in \mathbb{Z}^{n}} \cdots \sum_{s_{\alpha-\zeta} \in \mathbb{Z}^{n}} \prod_{i=1}^{\alpha-\zeta} c_{s_{i}}^{(\alpha-i)} \delta_{0,\left|\eta-\beta-\sum_{i=1}^{\alpha-\zeta} s_{i}\right|} .
$$

We now give further properties of the discrete fundamental solution. The following lemma is a simple consequence of Lemma 1 and the recurrence relations (11) and (12).

LEMMA 3. Under the assumptions of Lemma 2 we have

$$
\Gamma^{(\alpha, \beta, \zeta, \eta)} \geq 0, \quad \sum_{\eta \in \mathbb{Z}^{n}} \Gamma^{(\alpha, \beta, \zeta, \eta)}=1
$$

for $\alpha, \zeta=0,1, \ldots$ with $\alpha \geq \zeta$ and $\beta \in \mathbb{Z}^{n}$.

To obtain a priori estimates of the difference operators for the fundamental solution we will use the following lemma. The symbol $\lfloor r\rfloor$ stands for the integer part of $r \in \mathbb{R}$, i.e., the integer $k$ such that $k \leq r<k+1$.

LEMMA 4. If $0<b \leq 1 / 2$, then

$$
\sum_{k=0}^{i}\left(\begin{array}{l}
i \\
k
\end{array}\right)(1-2 b)^{i-k} b^{k}\left(\begin{array}{c}
k \\
\lfloor k / 2\rfloor
\end{array}\right) \leq \frac{1}{\sqrt{2 b(i+1)}}
$$

Proof. From a version of the Stirling formula [5] we have

$$
\sum_{k=0}^{i}\left(\begin{array}{l}
i \\
k
\end{array}\right)(1-2 b)^{i-k} b^{k}\left(\begin{array}{c}
k \\
\lfloor k / 2\rfloor
\end{array}\right) \leq \sum_{k=0}^{i}\left(\begin{array}{l}
i \\
k
\end{array}\right)(1-2 b)^{i-k} b^{k} \frac{2^{k}}{\sqrt{k+1}}
$$

By the Schwarz inequality, we get

$$
\begin{aligned}
& \sum_{k=0}^{i}\left(\begin{array}{l}
i \\
k
\end{array}\right)(1-2 b)^{i-k} b^{k} \frac{2^{k}}{\sqrt{k+1}} \\
& \quad \leq\left(\sum_{k=0}^{i}\left(\begin{array}{l}
i \\
k
\end{array}\right)(1-2 b)^{i-k}(2 b)^{k} \frac{1}{k+1}\right)^{1 / 2}\left(\sum_{k=0}^{i}\left(\begin{array}{l}
i \\
k
\end{array}\right)(1-2 b)^{i-k}(2 b)^{k}\right)^{1 / 2} \\
& \quad=\left(\sum_{k=0}^{i}\left(\begin{array}{l}
i \\
k
\end{array}\right)(1-2 b)^{i-k}(2 b)^{k} \frac{1}{k+1}\right)^{1 / 2} .
\end{aligned}
$$


Since

$$
\left(\begin{array}{l}
i \\
k
\end{array}\right) \frac{1}{k+1}=\left(\begin{array}{l}
i+1 \\
k+1
\end{array}\right) \frac{1}{i+1}
$$

we have

$$
\begin{aligned}
\sum_{k=0}^{i}\left(\begin{array}{c}
i \\
k
\end{array}\right)(1-2 b)^{i-k}(2 b)^{k} \frac{1}{k+1} & \leq \frac{1}{(i+1) 2 b} \sum_{k=0}^{i+1}\left(\begin{array}{c}
i+1 \\
k
\end{array}\right)(1-2 b)^{i+1-k}(2 b)^{k} \\
& =\frac{1}{(i+1) 2 b} .
\end{aligned}
$$

Hence the assertion follows.

Lemma 5. If Assumptions 1 and 2 are satisfied, and $h \in I_{C}$, then

$$
\sum_{\zeta=1}^{\alpha} \sum_{\eta \in \mathbb{Z}^{n}}\left|\Gamma^{\left(\alpha, \beta+e_{j}, \zeta, \eta\right)}-\Gamma^{\left(\alpha, \beta-e_{j}, \zeta, \eta\right)}\right| \leq 4 \frac{\sqrt{\alpha}}{\sqrt{2 \varepsilon}}
$$

where

$$
0<\varepsilon=\min _{j=1, \ldots, n, \alpha \geq 0} c_{+e_{j}}^{(\alpha)} .
$$

Proof. We prove this in three steps:

(a) some reductions and simplifications are made in

$$
\Delta \Gamma^{(\alpha, \zeta)}=\sum_{\eta \in \mathbb{Z}^{n}}\left|\Gamma^{\left(\alpha, \beta+e_{j}, \zeta, \eta\right)}-\Gamma^{\left(\alpha, \beta-e_{j}, \zeta, \eta\right)}\right|,
$$

(b) $\Delta \Gamma^{(\alpha, \zeta)}$ is shown to be an affine and decreasing function in the variables $c_{ \pm e_{j}}^{(\gamma)}(\zeta \leq \gamma<\alpha)$

(c) the assertion of Lemma 5 is deduced from Assumption 1 and Lemma 4.

STEP (a). Without loss of generality we assume that $j=1$, hence

$$
\Delta \Gamma^{(\alpha, \zeta)}=\sum_{\eta \in \mathbb{Z}^{n}}\left|\Gamma^{\left(\alpha, \beta+e_{1}, \zeta, \eta\right)}-\Gamma^{\left(\alpha, \beta-e_{1}, \zeta, \eta\right)}\right| .
$$

Thus from Remark 1 we have

$$
\begin{aligned}
\Delta \Gamma^{(\alpha, \zeta)}= & \sum_{\eta \in \mathbb{Z}^{n}} \mid \sum_{s_{1} \in \mathbb{Z}^{n}} \ldots \sum_{s_{\alpha-\zeta} \in \mathbb{Z}^{n}} \prod_{i=1}^{\alpha-\zeta} c_{s_{i}}^{(\alpha-i)} \delta_{0,\left|\eta-\beta-e_{1}-\sum_{i=1}^{\alpha-\zeta} s_{i}\right|} \\
& -\sum_{s_{1} \in \mathbb{Z}^{n}} \ldots \sum_{s_{\alpha-\zeta} \in \mathbb{Z}^{n}} \prod_{i=1}^{\alpha-\zeta} c_{s_{i}}^{(\alpha-i)} \delta_{0,\left|\eta-\beta+e_{1}-\sum_{i=1}^{\alpha-\zeta} s_{i}\right|} \mid .
\end{aligned}
$$

Because each multiindex in $\mathbb{Z}^{n}$ can be decomposed as $m e_{1}+\eta$, where $\eta_{1}=0$ and $m \in \mathbb{Z}$, we rearrange the above formula as follows: 
(14) $\Delta \Gamma^{(\alpha, \zeta)}=$

$$
\sum_{m \in \mathbb{Z}} \sum_{\eta \in \mathbb{Z}^{n}, \eta_{1}=0}\left|\sum_{\sum_{i}} \prod_{s_{i}=(m+1) e_{1}+\eta} \prod_{i=1}^{\alpha-\zeta} c_{s_{i}}^{(\alpha-i)}-\sum_{\sum_{i}} \sum_{s_{i}=(m-1) e_{1}+\eta} \prod_{i=1}^{\alpha-\zeta} c_{s_{i}}^{(\alpha-i)}\right| .
$$

Observe that

$$
\sum_{\sum_{i}} \prod_{s_{i}=(m+1) e_{1}+\eta}^{\alpha-\zeta} c_{s_{i}}^{(\alpha-i)}-\sum_{\sum_{i} s_{i}=(m-1) e_{1}+\eta} \prod_{i=1}^{\alpha-\zeta} c_{s_{i}}^{(\alpha-i)} \geq 0
$$

iff $|m+1| \leq|m-1|$, i.e., $m \leq 0$. This follows from the fact that $c_{s}^{(\gamma)}=$ $c_{-s}^{(\gamma)}$. Thus for all $m \leq 0$, there is an injection from the set of multiindices $\left(s_{1}, \ldots, s_{\alpha-\zeta}\right)$ for which

$$
\sum_{i} s_{i}=(m-1) e_{1}+\eta, \quad \eta_{1}=0
$$

to the set of multiindices $\left(\bar{s}_{1}, \ldots, \bar{s}_{\alpha-\zeta}\right)$ for which

$$
\sum_{i} \bar{s}_{i}=(m+1) e_{1}+\eta, \quad \eta_{1}=0 .
$$

A similar (reverse) injection can be found for all $m>0$. The construction of the injection is based on a proper replacement of some coefficients $c_{s_{l}}^{(\alpha-l)}=c_{+e_{1}}^{(\alpha-l)}$ by $c_{\bar{s}_{l}}^{(\alpha-l)}=c_{-e_{1}}^{(\alpha-l)}$. In the case of $m=0$, the above injection $\left(s_{1}, \ldots, s_{\alpha-\zeta}\right) \mapsto\left(\bar{s}_{1}, \ldots, \bar{s}_{\alpha-\zeta}\right)$ becomes a bijection. Hence by (14) and (15) we have

$$
\begin{aligned}
\Delta \Gamma^{(\alpha, \zeta)}= & \sum_{\eta \in \mathbb{Z}^{n}, \eta_{1}=0}\left(\sum_{\sum_{i}} \prod_{s_{i}=-e_{1}+\eta} \prod_{i=1}^{\alpha-\zeta} c_{s_{i}}^{(\alpha-i)}+\sum_{\sum_{i} s_{i}=\eta} \prod_{i=1}^{\alpha-\zeta} c_{s_{i}}^{(\alpha-i)}\right. \\
& \left.+\sum_{\sum_{i} s_{i}=\eta} \prod_{i=1}^{\alpha-\zeta} c_{s_{i}}^{(\alpha-i)}+\sum_{\sum_{i}} \prod_{s_{i}=+e_{1}+\eta}^{\alpha-\zeta} c_{i=1}^{(\alpha-i)}\right)
\end{aligned}
$$

because all remaining terms cancel each other. Since $c_{+e_{1}}^{(\gamma)}=c_{-e_{1}}^{(\gamma)}$ we can rewrite the above formula as

$$
\Delta \Gamma^{(\alpha, \zeta)}=2 \sum_{\eta \in \mathbb{Z}^{n}, \eta_{1}=0}\left(\sum_{\sum_{i} s_{i}=\eta} \prod_{i=1}^{\alpha-\zeta} c_{s_{i}}^{(\alpha-i)}+\sum_{\sum_{i}} \sum_{s_{i}=+e_{1}+\eta} \prod_{i=1}^{\alpha-\zeta} c_{s_{i}}^{(\alpha-i)}\right)
$$

or $\Delta \Gamma^{(\alpha, \zeta)}=\Delta \Gamma_{0}^{(\alpha, \zeta)}+\Delta \Gamma_{+1}^{(\alpha, \zeta)}$, where

$$
\Delta \Gamma_{m}^{(\alpha, \zeta)}=2 \sum_{\eta \in \mathbb{Z}^{n}, \eta_{1}=0} \sum_{\sum_{i}} \prod_{s_{i}=m e_{1}+\eta}^{\alpha-\zeta} c_{i=1}^{(\alpha-i)}, \quad m \in \mathbb{Z} .
$$


Hence we have to consider only sequences $\left(s_{1}, \ldots, s_{\alpha-\zeta}\right)$ such that

$$
\left(\sum_{i=1}^{\alpha-\zeta} s_{i}=0+\eta, \eta_{1}=0\right) \quad \text { or } \quad\left(\sum_{i=1}^{\alpha-\zeta} s_{i}=+e_{1}+\eta, \eta_{1}=0\right) .
$$

The sequences will be classified according to the appearance of $s_{i}=+e_{1}$, $s_{i}=-e_{1}$ and other indices. Let $J=\{1, \ldots, \alpha-\zeta\}$. Denote by $A$ and $B$ any disjoint subsets of $J$ whose cardinalities satisfy either $\# A=\# B$ or $\# B=\# A-1$. The set $A$ is related to indices $s=+e_{1}$, while the set $B$ to indices $s=-e_{1}$, so condition (18) is met. Since $A \cap B=\emptyset$ and $A \cup B \subset J$ it is obvious that $2 \# A \leq \# J+1$. We have $c_{+e_{1}}^{(\gamma)}=c_{-e_{1}}^{(\gamma)}$ and $A \cap B=\emptyset$, so it will cause no confusion if we use $c_{ \pm e_{1}}^{(\gamma)}$ instead of $c_{+e_{1}}^{(\gamma)}$ and $c_{-e_{1}}^{(\gamma)}$ to simplify some of the formulas. For any fixed $k \in J$ and any $\left(s_{i}\right)_{i \neq k}$, the sum over $s_{k} \in \mathbb{Z}^{n}$, $s_{k} \neq \pm e_{1}$ (part of the sum over $\eta$ in (16)), yields $\left(1-2 c_{ \pm e_{1}}^{(\alpha-k)}\right) \prod_{i \in J, i \neq k} c_{s_{i}}^{(\alpha-i)}$. Thus we can represent $\Delta \Gamma^{(\alpha, \zeta)}$ as follows:

$$
\Delta \Gamma^{(\alpha, \zeta)}=2 \sum_{\substack{A, B \subset J \\ A \cap B=\emptyset \\ A-\# B \in\{0,1\}}} \prod_{k \in A} c_{+e_{1}}^{(\alpha-k)} \prod_{k \in B} c_{-e_{1}}^{(\alpha-k)} \prod_{k \in J \backslash(A \cup B)}\left(1-2 c_{ \pm e_{1}}^{(\alpha-k)}\right) .
$$

This finishes the proof of step (a).

STEP (b). It follows from (19) that $\Delta \Gamma^{(\alpha, \zeta)}$ is an affine function with respect to $c_{ \pm e_{1}}^{(\alpha)}$ for all $k \in J$. Indeed, this is straightforward because $k$ belongs either to $A, B$ or $J \backslash(A \cup B)$. Now we show by induction on $\alpha>\zeta$ that $\Delta \Gamma^{(\alpha, \zeta)}$ is decreasing with respect to all arguments $c_{ \pm e_{1}}^{(\gamma)}$. This is obvious for $\alpha=\zeta+1$ (see (16) for $\left.\Delta \Gamma^{(\zeta+1, \zeta)}\right)$. Assume that $\Delta \Gamma^{(\alpha, \zeta)}$ decreases in all $c_{ \pm e_{1}}^{(\gamma)}$. We will prove it for $\Delta \Gamma^{(\alpha+1, \zeta)}$. It suffices to consider $c_{ \pm e_{1}}^{(\alpha)}$, because the proof for arbitrary $\zeta \leq \gamma \leq \alpha$ is analogous. We will show that

$$
\Delta \Gamma^{(\alpha, \zeta)}\left(1-c_{ \pm e_{1}}^{(\alpha)}\right) \leq \Delta \Gamma^{(\alpha+1, \zeta)} \leq \Delta \Gamma^{(\alpha, \zeta)}-c_{ \pm e_{1}}^{(\alpha)} \prod_{i \in J}\left(1-2 c_{ \pm e_{1}}^{(\alpha-i)}\right),
$$

i.e., $\Delta \Gamma^{(\alpha+1, \zeta)}$ is an affine function, bounded by two affine decreasing functions with the same value at 0 . Thus $\Delta \Gamma^{(\alpha+1, \zeta)}$ is decreasing in $c_{ \pm e_{1}}^{(\alpha)}$.

Now we prove (20). By multiplying $c_{s}^{(\alpha)}$ by appropriate $\Delta \Gamma_{m}^{(\alpha, \zeta)}$ (see (17) and (18)) and summing them over $s \in \mathbb{Z}^{n}$, we get the recurrence formula for $\Delta \Gamma^{(\alpha+1, \zeta)}$ :

$$
\begin{aligned}
\Delta \Gamma^{(\alpha+1, \zeta)}= & \left(1-c_{+e_{1}}^{(\alpha)}-c_{-e_{1}}^{(\alpha)}\right) \Delta \Gamma_{0}^{(\alpha, \zeta)}+c_{+e_{1}}^{(\alpha)} \Delta \Gamma_{0}^{(\alpha, \zeta)}+c_{-e_{1}}^{(\alpha)} \Delta \Gamma_{+2}^{(\alpha, \zeta)} \\
& +\left(1-c_{+e_{1}}^{(\alpha)}-c_{-e_{1}}^{(\alpha)}\right) \Delta \Gamma_{+1}^{(\alpha, \zeta)}+c_{+e_{1}}^{(\alpha)} \Delta \Gamma_{-1}^{(\alpha, \zeta)}+c_{-e_{1}}^{(\alpha)} \Delta \Gamma_{+1}^{(\alpha, \zeta)} \\
= & \Delta \Gamma^{(\alpha, \zeta)}-c_{ \pm e_{1}}^{(\alpha)}\left(\Delta \Gamma_{0}^{(\alpha, \zeta)}-\Delta \Gamma_{+2}^{(\alpha, \zeta)}\right) .
\end{aligned}
$$

By omitting in (21) the nonnegative terms $c_{-e_{1}}^{(\alpha)} \Delta \Gamma_{+1}^{(\alpha, \zeta)}$ and $c_{-e_{1}}^{(\alpha)} \Delta \Gamma_{+2}^{(\alpha, \zeta)}$, we 
get the lower bound in (20). Since $\Delta \Gamma_{0}^{(\alpha, \zeta)}$ contains sequences of coefficients without any indices $s_{i}= \pm e_{1}$, which sum to $\prod_{i \in J}\left(1-2 c_{ \pm e_{1}}^{(\alpha-i)}\right)$, we get the stronger version of (15), namely $\Delta \Gamma_{0}^{(\alpha, \zeta)} \geq \Delta \Gamma_{+2}^{(\alpha, \zeta)}+\prod_{i \in J}\left(1-2 c_{ \pm e_{1}}^{(\alpha)}\right)$. This gives the upper bound in (20). This finishes the proof of (b).

STEP (c). It follows from Assumption 1 that $c_{ \pm e_{1}}^{(\gamma)} \geq \varepsilon>0$. From step (b) and formula (19) we have the inequality

$$
\Delta \Gamma^{(\alpha, \zeta)} \leq 2 \sum_{\substack{A, B \subset J \\ A \cap B=\emptyset \\ A A-\# B \in\{0,1\}}} \prod_{k \in A \cup B} \varepsilon \prod_{k \in J \backslash(A \cup B)}(1-2 \varepsilon) .
$$

Let $i=\#(A \cup B)$. Observe that $0 \leq i \leq \alpha-\zeta$. Thus by basic combinatorics we have

$$
\Delta \Gamma^{(\alpha, \zeta)} \leq 2 \sum_{i=0}^{\alpha-\zeta}\left(\begin{array}{c}
\alpha-\zeta \\
i
\end{array}\right)\left(\begin{array}{c}
i \\
\lfloor i / 2\rfloor
\end{array}\right)(1-2 \varepsilon)^{\alpha-\zeta-i} \varepsilon^{i} .
$$

Applying Lemma 4, we get

$$
\Delta \Gamma^{(\alpha, \zeta)} \leq 2 \frac{1}{\sqrt{2 \varepsilon(\alpha-\zeta+1)}}
$$

Finally, summing over $\zeta$ in $(22)$ yields

$$
\sum_{\zeta=1}^{\alpha} \sum_{\eta \in \mathbb{Z}^{n}}\left|\Gamma^{\left(\alpha, \beta+e_{1}, \zeta, \eta\right)}-\Gamma^{\left(\alpha, \beta-e_{1}, \zeta, \eta\right)}\right| \leq 4 \frac{\sqrt{\alpha}}{\sqrt{2 \varepsilon}} .
$$

Hence the proof of Lemma 5 is complete.

Now from Lemmas 2, 3 and 5 we get

Corollary 1.

$$
\left|\delta_{j} u^{(\alpha+1, \beta)}\right| \leq\left\|\delta_{j} u\right\|_{\infty}(0)+C_{j} \frac{\sqrt{2 t^{\alpha+1}}}{\sqrt{\varepsilon}}\|g\|_{\infty}(\alpha) .
$$

2. Stability and convergence. The defect of scheme (4)-(5) will be defined by

$$
\Theta[u, h]^{(\alpha, \beta)}=\delta_{0}^{+} u^{(\alpha, \beta)}-\sum_{k=1}^{n} a_{k}^{(\alpha)} \delta_{k k} u^{(\alpha, \beta)}-f[u]^{(\alpha, \beta)} .
$$

If $\Theta[u, h]^{(\alpha, \beta)} \equiv 0$, then $\left\{u^{(\alpha, \beta)}\right\}$ is a solution of scheme (4)-(5). We formulate sufficient consistency and stability conditions. All supremum norms will be denoted by $\|\cdot\|_{\infty}$.

Assumption 3. There are constants $L_{1}, L_{2} \in \mathbb{R}_{+}$such that

$$
\left|f_{h}(t, x, p, q)-f_{h}(t, x, \widetilde{p}, \widetilde{q})\right| \leq L_{1}\|p-\widetilde{p}\|_{\infty}+L_{2}\|q-\widetilde{q}\|_{\infty}
$$

for all $(t, x, p, q),(t, x, \widetilde{p}, \widetilde{q}) \in E_{h}^{+} \times{ }^{B_{h}} \mathbb{R} \times\left({ }^{B_{h}} \mathbb{R}\right)^{n}$. 
The above assumption is similar to the third part of Assumption $\mathrm{H}\left[f_{h}, \sigma_{h}\right]$ in [2].

Assumption 4. The discrete function $f_{h}=f[u]^{(\alpha, \beta)}$ (see (6)) satisfies

$$
\left|f_{h}\left(t^{\alpha}, x^{\beta}, u_{[\alpha, \beta]},(\delta u)_{[\alpha, \beta]}\right)-f\left(t^{\alpha}, x^{\beta}, u_{\left(t^{\alpha}, x^{\beta}\right)},\left(\partial_{x} u\right)_{\left(t^{\alpha}, x^{\beta}\right)}\right)\right| \leq C\|h\|_{\infty}
$$

for all $\left(t^{\alpha}, x^{\beta}\right) \in E_{h}^{+}$, with a constant $C$ depending on $u, \partial_{x_{1}} u, \ldots, \partial_{x_{n}} u$, where $u: E_{0} \cup E \rightarrow \mathbb{R}$.

It can be shown (see [3]) that there exists an interpolation operator $T_{h}$ such that $T_{h} w \in C(B, \mathbb{R})$ for arbitrary $w: B_{h} \rightarrow \mathbb{R}$, and there exist constants $\breve{C}, \bar{C}>0$ such that

$$
\left|T_{h}\left(v_{\mid B_{h}}\right)(t, x)-v(t, x)\right| \leq \breve{C}\|h\|_{\infty} \quad \text { for } v \in C^{1}(B)
$$

and

$$
\left\|T_{h} w-T_{h} \bar{w}\right\|_{\infty} \leq \bar{C}\|w-\bar{w}\|_{\infty} \quad \text { for } w, \bar{w}: B_{h} \rightarrow \mathbb{R} .
$$

If we define

$$
f_{h}(t, x, p, q)=f\left(t, x, T_{h} p, T_{h} q\right)
$$

for $(t, x, p, q) \in E_{h}^{+} \times{ }^{B_{h}} \mathbb{R} \times\left({ }^{B_{h}} \mathbb{R}\right)^{n}$, then Assumption 4 is satisfied, provided that $f=f(t, x, p, q)$ fulfills the Lipschitz condition with respect to $p, q$ and $u \in C^{2}\left(E_{0} \cup E, \mathbb{R}\right)$.

Assumption 5. There exists a unique solution $u \in C^{2,3}\left(E_{0} \cup E, \mathbb{R}\right)$ of the Cauchy problem (1), (2).

Lemma 6 (consistency). Suppose that Assumptions 4 and 5 are satisfied and $\left\|\phi^{(\alpha, \beta)}-\bar{\phi}^{(\alpha, \beta)}\right\|_{\infty} \rightarrow 0$ as $\|h\|_{\infty} \rightarrow 0$. Then scheme (4), (5) is consistent with the Cauchy problem (1), (2) on its solution $u \in C^{2,3}\left(E_{0} \cup E, \mathbb{R}\right)$.

Proof. The consistency is obtained by using the Taylor expansions at nodal points (an analogous result was proved in [6]).

Lemma 7 (stability). If $u, v: \widetilde{E}_{h} \rightarrow \mathbb{R}$ and Assumption 3 is satisfied, and

$$
\begin{gathered}
\left|u^{(\alpha, \beta)}-v^{(\alpha, \beta)}\right| \leq \check{C}\|h\|_{\infty} \quad \text { on } E_{h}^{0}, \\
\left|\delta_{j}(u-v)^{(\alpha, \beta)}\right| \leq \widetilde{C}\|h\|_{\infty} \quad \text { on } E_{h}^{0}, j=1, \ldots, n, \\
\Theta[u, h]^{(\alpha, \beta)}=0, \quad\left|\Theta[v, h]^{(\alpha, \beta)}\right| \leq \bar{C}\|h\|_{\infty} \quad \text { on } E_{h}^{+}, \\
L_{1} d+\widehat{C} \sqrt{2 / \varepsilon} \sqrt{d} L_{2}<1, \quad \text { where } \quad \widehat{C}=\max _{j \in\{1, \ldots, n\}} C_{j}
\end{gathered}
$$

then

$$
\sup _{\alpha, \beta}\left\|\left(u^{(\alpha, \beta)}-v^{(\alpha, \beta)}, \delta(u-v)^{(\alpha, \beta)}\right)\right\|_{\infty} \rightarrow 0 \quad \text { as }\|h\|_{\infty} \rightarrow 0 .
$$


Proof. Set

$$
\omega^{(\alpha, \beta)}=u^{(\alpha, \beta)}-v^{(\alpha, \beta)}, \quad \gamma^{(\alpha, \beta)}=f[u]^{(\alpha, \beta)}-f[v]^{(\alpha, \beta)}-\Theta[v, h]^{(\alpha, \beta)} .
$$

From Lemma 2 and Corollary 1 it follows that

$$
\begin{aligned}
& \|\omega\|_{\infty}(\alpha+1) \leq\|\omega\|_{\infty}(0)+t^{\alpha+1}\|\gamma\|_{\infty}(\alpha) \\
& \quad \leq\|\omega\|_{\infty}(0)+t^{\alpha+1}\left\{L_{1}\|\omega\|_{\infty}(\alpha)+L_{2}\|\delta \omega\|_{\infty}(\alpha)\right\}+t^{\alpha+1} \bar{C}\|h\|_{\infty}
\end{aligned}
$$

and

$$
\begin{aligned}
\|\delta \omega\|_{\infty}(\alpha+1) \leq & \|\delta \omega\|_{\infty}(0)+\widehat{C} \sqrt{2 / \varepsilon} \sqrt{t^{\alpha+1}}\|\gamma\|_{\infty}(\alpha) \\
\leq & \|\delta \omega\|_{\infty}(0)+\widehat{C} \sqrt{2 / \varepsilon} \sqrt{t^{\alpha+1}}\left\{L_{1}\|\omega\|_{\infty}(\alpha)+L_{2}\|\delta \omega\|_{\infty}(\alpha)\right\} \\
& +\bar{C} \widehat{C} \sqrt{2 / \varepsilon} \sqrt{t^{\alpha+1}}\|h\|_{\infty} .
\end{aligned}
$$

Observe that

$$
L_{1} t^{\alpha+1}+\widehat{C} \sqrt{2 / \varepsilon} \sqrt{t^{\alpha+1}} L_{2} \leq \kappa<1 \quad \text { for } 0 \leq t^{\alpha+1} \leq d,
$$

where $\kappa:=L_{1} d+\widehat{C} \sqrt{2 / \varepsilon} \sqrt{d} L_{2}$. If we set

$$
\zeta^{(\alpha)}=L_{1}\|\omega\|_{\infty}(\alpha)+L_{2}\|\delta \omega\|_{\infty}(\alpha)
$$

then

$$
\begin{aligned}
\zeta^{(\alpha+1) \leq} & \zeta^{(0)}+\left(L_{1} t^{\alpha+1}+\widehat{C} \sqrt{2 / \varepsilon} \sqrt{t^{\alpha+1}} L_{2}\right) \zeta^{(\alpha)} \\
& +\left(L_{1} t^{\alpha+1}+\widehat{C} \sqrt{2 / \varepsilon} \sqrt{t^{\alpha+1}} L_{2}\right) \bar{C}\|h\|_{\infty} .
\end{aligned}
$$

Hence

$$
\|\zeta\|_{\infty} \leq \frac{1}{1-\kappa}\left(\widetilde{C}\|h\|_{\infty}+\check{C}\|h\|_{\infty}\right)+\frac{\kappa}{1-\kappa} \bar{C}\|h\|_{\infty} .
$$

Thus $\|\zeta\|_{\infty}$ tends to 0 as $\|h\|_{\infty} \rightarrow 0$, which means that so do $\|\omega\|_{\infty}$ and $\|\delta \omega\|_{\infty}$.

THEOREM 1 (convergence). Suppose that Assumptions 3-5 are satisfied, $L_{1} d+\widehat{C} \sqrt{2 / \varepsilon} \sqrt{d} L_{2}<1$ and

$$
\|\left(\phi^{(\alpha, \beta)}-\bar{\phi}^{(\alpha, \beta)}, \delta(\phi-\bar{\phi})^{(\alpha, \beta)} \|_{\infty}=O\left(\|h\|_{\infty}\right)\right.
$$

Then the solutions of scheme (4), (5) converge to the unique solution of the differential-functional problem (1), (2).

Proof. The assertion follows from Lemmas 6 and 7 .

REMARK 2. The assumption on the class of the solution of (1), (2) in Lemma 6 and Assumption 5 can be weakened to $C^{1,2}$, but in that case we get in Theorem 1 only $\|\zeta\|_{\infty}=o(1)$ instead of $\|\zeta\|_{\infty}=O\left(\|h\|_{\infty}\right)$. If we assume class $C^{2,4}$ and the estimate by $O\left(\|h\|_{\infty}^{2}\right)$ in Assumption 4 and (23), then we get $\|\zeta\|_{\infty}=O\left(h_{0}+\left\|h^{\prime}\right\|_{\infty}^{2}\right)=O\left(\left\|h^{\prime}\right\|_{\infty}^{2}\right)$ (see [3]). 
3. Numerical experiments. Fix $n=2$ and consider the equations

(25) $\partial_{t} u\left(t, x_{1}, x_{2}\right)-\sum_{k=1}^{2} a_{k}(t) \partial_{x_{k} x_{k}} u\left(t, x_{1}, x_{2}\right)$

$$
\partial_{t} u\left(t, x_{1}, x_{2}\right)-\sum_{k=1}^{2} a_{k}(t) \partial_{x_{k} x_{k}} u\left(t, x_{1}, x_{2}\right)=f_{1}\left(t, x_{1}, x_{2}\right),
$$

$$
=\sin \left(u\left(t, x_{1}+\sin \left(x_{1}\right), x_{2}\right)\right)+f_{2}\left(t, x_{1}, x_{2}\right),
$$

(26) $\partial_{t} u\left(t, x_{1}, x_{2}\right)-\sum_{k=1}^{2} a_{k}(t) \partial_{x_{k} x_{k}} u\left(t, x_{1}, x_{2}\right)$

$$
=\sin \left(\int_{x_{1}-1}^{x_{1}+1} u\left(t, s, x_{2}\right) d s\right)+f_{3}\left(t, x_{1}, x_{2}\right),
$$

$$
\begin{aligned}
\partial_{t} u\left(t, x_{1}, x_{2}\right)-\sum_{k=1}^{2} a_{k}(t) \partial_{x_{k} x_{k}} u\left(t, x_{1}, x_{2}\right) & \\
= & \sin \left(\partial_{x_{1}} u\left(t, x_{1}, x_{2}\right)\right)+f_{4}\left(t, x_{1}, x_{2}\right),
\end{aligned}
$$

(28) $\partial_{t} u\left(t, x_{1}, x_{2}\right)-\sum_{k=1}^{2} a_{k}(t) \partial_{x_{k} x_{k}} u\left(t, x_{1}, x_{2}\right)$

$$
=\sin \left(\partial_{x_{1}} u\left(t, x_{1}+\sin \left(x_{1}\right), x_{2}\right)\right)+f_{5}\left(t, x_{1}, x_{2}\right),
$$

(29) $\partial_{t} u\left(t, x_{1}, x_{2}\right)-\sum_{k=1}^{2} a_{k}(t) \partial_{x_{k} x_{k}} u\left(t, x_{1}, x_{2}\right)$

$$
=\sin \left(\partial_{x_{1}} u\left(t-\tau_{0}, x_{1}, x_{2}\right)\right)+f_{6}\left(t, x_{1}, x_{2}\right),
$$

(30) $\partial_{t} u\left(t, x_{1}, x_{2}\right)-\sum_{k=1}^{2} a_{k}(t) \partial_{x_{k} x_{k}} u\left(t, x_{1}, x_{2}\right)$

$$
=\sin \left(u\left(t-\tau_{0}, x_{1}, x_{2}\right)\right)+f_{7}\left(t, x_{1}, x_{2}\right),
$$

(31) $\partial_{t} u\left(t, x_{1}, x_{2}\right)-\sum_{k=1}^{2} a_{k}(t) \partial_{x_{k} x_{k}} u\left(t, x_{1}, x_{2}\right)$

$$
=\sin \left(\int_{t-\tau_{0}}^{t} \int_{x_{1}-1}^{x_{1}+1} u\left(s, z, x_{2}\right) d z d s\right)+f_{8}\left(t, x_{1}, x_{2}\right) .
$$

Consider two sample functions $\left(a_{1}(t), a_{2}(t)\right)$ :

- case I: $a_{1}(t)=a_{2}(t)=2+\sin (t)$,

- case II: $a_{1}(t)=a_{2}(t)= \begin{cases}1+t, & t \in[0,0.5), \\ 2-t, & t \in[0.5,1] .\end{cases}$ 
We have performed numerical experiments for equations (24)-(31) in both cases. The results have been compared with the prescribed solution

$$
u(t, x, y)=\cos \left(t \sin \left(t+x_{1}+x_{2}\right)\right) .
$$

The right-hand sides $f_{1}\left(t, x_{1}, x_{2}\right), \ldots, f_{8}\left(t, x_{1}, x_{2}\right)$ are defined so as to make $u$ a solution. Since the computations require a large amount of time and computer memory (especially for small $h_{0}$ ), the domain considered in the computations is restricted to $[0,1] \times[-11,11]^{2}$ with boundary values equal to the initial values at $t=0$. This may cause large errors near the boundary, so we present the maximal errors on a smaller domain, namely $[0,1] \times[-1,1]^{2}$. The maximal errors on $[0,1] \times[-1,1]^{2}$ are presented in Tables $1-2$ and $3-4$, respectively. The errors for analogous tests on a smaller domain, e.g. $[0,1] \times[-6,6]^{2}$ turn out to be larger than those listed above. All numerical experiments confirm convergence of a discrete function to the exact solution.

Acknowledgements. We are grateful to the reviewer for his valuable remarks, which improved the entire paper.

Table 1. The maximal error on $[0,1] \times[-1,1]^{2}, \widetilde{h}=h_{1}=h_{2}=1 / n$ and $h_{0}=1 /\left(12 n^{2}+1\right)$

\begin{tabular}{lcccccc}
\hline$\widetilde{h}$ & \multicolumn{2}{c}{$1 / 5$} & \multicolumn{2}{c}{$1 / 10$} & \multicolumn{2}{c}{$1 / 20$} \\
\hline$t$-interval & {$[0,0.5]$} & {$[0,1]$} & {$[0,0.5]$} & {$[0,1]$} & {$[0,0.5]$} & {$[0,1]$} \\
\hline$(24)$ & $1.06 \mathrm{e}-03$ & $3.85 \mathrm{e}-03$ & $2.66 \mathrm{e}-04$ & $9.58 \mathrm{e}-04$ & $6.66 \mathrm{e}-05$ & $2.41 \mathrm{e}-04$ \\
$(25)$ & $1.14 \mathrm{e}-03$ & $4.20 \mathrm{e}-03$ & $2.85 \mathrm{e}-04$ & $1.05 \mathrm{e}-03$ & $7.13 \mathrm{e}-05$ & $2.63 \mathrm{e}-04$ \\
$(26)$ & $9.87 \mathrm{e}-04$ & $3.66 \mathrm{e}-03$ & $2.47 \mathrm{e}-04$ & $9.11 \mathrm{e}-04$ & $6.19 \mathrm{e}-05$ & $2.29 \mathrm{e}-04$ \\
$(27)$ & $1.09 \mathrm{e}-03$ & $3.88 \mathrm{e}-03$ & $2.73 \mathrm{e}-04$ & $9.79 \mathrm{e}-04$ & $6.86 \mathrm{e}-05$ & $2.45 \mathrm{e}-04$ \\
$(28)$ & $1.15 \mathrm{e}-03$ & $3.96 \mathrm{e}-03$ & $2.89 \mathrm{e}-04$ & $9.85 \mathrm{e}-04$ & $7.20 \mathrm{e}-05$ & $2.50 \mathrm{e}-04$ \\
$(29)\left(\tau_{0}=0.5\right)$ & $1.06 \mathrm{e}-03$ & $3.83 \mathrm{e}-03$ & $2.66 \mathrm{e}-04$ & $9.53 \mathrm{e}-04$ & $6.66 \mathrm{e}-05$ & $2.39 \mathrm{e}-04$ \\
$(29)\left(\tau_{0}=0.25\right)$ & $1.06 \mathrm{e}-03$ & $3.77 \mathrm{e}-03$ & $2.66 \mathrm{e}-04$ & $9.39 \mathrm{e}-04$ & $6.66 \mathrm{e}-05$ & $2.36 \mathrm{e}-04$ \\
$(30)\left(\tau_{0}=0.5\right)$ & $1.06 \mathrm{e}-03$ & $3.81 \mathrm{e}-03$ & $2.66 \mathrm{e}-04$ & $9.49 \mathrm{e}-04$ & $6.66 \mathrm{e}-05$ & $2.38 \mathrm{e}-04$ \\
$(30)\left(\tau_{0}=0.25\right)$ & $1.06 \mathrm{e}-03$ & $3.76 \mathrm{e}-03$ & $2.65 \mathrm{e}-04$ & $9.35 \mathrm{e}-04$ & $6.64 \mathrm{e}-05$ & $2.35 \mathrm{e}-04$ \\
$(31)\left(\tau_{0}=0.5\right)$ & $8.41 \mathrm{e}-04$ & $1.73 \mathrm{e}-03$ & $2.11 \mathrm{e}-04$ & $4.35 \mathrm{e}-04$ & $5.30 \mathrm{e}-05$ & $1.09 \mathrm{e}-04$ \\
$(31)\left(\tau_{0}=0.25\right)$ & $8.42 \mathrm{e}-04$ & $1.73 \mathrm{e}-03$ & $2.11 \mathrm{e}-04$ & $4.36 \mathrm{e}-04$ & $5.30 \mathrm{e}-05$ & $1.09 \mathrm{e}-04$ \\
\hline
\end{tabular}

Table 2. The maximal error for $u_{x}(t, x, y)$ with the same parameters as in Table 1

\begin{tabular}{lcccccc}
\hline$\widetilde{h}$ & \multicolumn{2}{c}{$1 / 5$} & \multicolumn{2}{c}{$1 / 10$} & \multicolumn{2}{c}{$1 / 20$} \\
\hline$t$-interval & {$[0,0.5]$} & {$[0,1]$} & {$[0,0.5]$} & {$[0,1]$} & {$[0,0.5]$} & {$[0,1]$} \\
\hline$(27)$ & $2.02 \mathrm{e}-03$ & $7.20 \mathrm{e}-03$ & $5.08 \mathrm{e}-04$ & $1.80 \mathrm{e}-03$ & $1.28 \mathrm{e}-04$ & $4.50 \mathrm{e}-04$ \\
$(28)$ & $2.30 \mathrm{e}-03$ & $7.98 \mathrm{e}-03$ & $5.85 \mathrm{e}-04$ & $2.02 \mathrm{e}-03$ & $1.47 \mathrm{e}-04$ & $5.06 \mathrm{e}-04$ \\
$(29)\left(\tau_{0}=0.5\right)$ & $2.02 \mathrm{e}-03$ & $7.13 \mathrm{e}-03$ & $5.06 \mathrm{e}-04$ & $1.78 \mathrm{e}-03$ & $1.27 \mathrm{e}-04$ & $4.45 \mathrm{e}-04$ \\
$(29)\left(\tau_{0}=0.25\right)$ & $2.01 \mathrm{e}-03$ & $7.27 \mathrm{e}-03$ & $5.05 \mathrm{e}-04$ & $1.81 \mathrm{e}-03$ & $1.26 \mathrm{e}-04$ & $4.54 \mathrm{e}-04$ \\
\hline
\end{tabular}


Table 3. The maximal error on $[0,1] \times[-1,1]^{2}, \widetilde{h}=h_{1}=h_{2}=1 / n$ and $h_{0}=1 /\left(6 n^{2}+1\right)$

\begin{tabular}{lcccccc}
\hline$\widetilde{h}$ & \multicolumn{2}{c}{$1 / 5$} & \multicolumn{2}{c}{$1 / 10$} & \multicolumn{2}{c}{$1 / 20$} \\
\hline$t$-interval & {$[0,0.5]$} & {$[0,1]$} & {$[0,0.5]$} & {$[0,1]$} & {$[0,0.5]$} & {$[0,1]$} \\
\hline$(24)$ & $1.34 \mathrm{e}-03$ & $4.34 \mathrm{e}-03$ & $3.37 \mathrm{e}-04$ & $1.08 \mathrm{e}-03$ & $8.47 \mathrm{e}-05$ & $2.71 \mathrm{e}-04$ \\
$(25)$ & $1.48 \mathrm{e}-03$ & $5.05 \mathrm{e}-03$ & $3.73 \mathrm{e}-04$ & $1.26 \mathrm{e}-03$ & $9.36 \mathrm{e}-05$ & $3.16 \mathrm{e}-04$ \\
$(26)$ & $1.20 \mathrm{e}-03$ & $3.99 \mathrm{e}-03$ & $3.02 \mathrm{e}-04$ & $9.95 \mathrm{e}-04$ & $7.58 \mathrm{e}-05$ & $2.49 \mathrm{e}-04$ \\
$(27)$ & $1.46 \mathrm{e}-03$ & $4.79 \mathrm{e}-03$ & $3.67 \mathrm{e}-04$ & $1.20 \mathrm{e}-03$ & $9.22 \mathrm{e}-05$ & $2.99 \mathrm{e}-04$ \\
$(28)$ & $1.47 \mathrm{e}-03$ & $4.69 \mathrm{e}-03$ & $3.74 \mathrm{e}-04$ & $1.18 \mathrm{e}-03$ & $9.32 \mathrm{e}-05$ & $2.96 \mathrm{e}-04$ \\
$(29)\left(\tau_{0}=0.5\right)$ & $1.34 \mathrm{e}-03$ & $4.34 \mathrm{e}-03$ & $3.37 \mathrm{e}-04$ & $1.09 \mathrm{e}-03$ & $8.47 \mathrm{e}-05$ & $2.72 \mathrm{e}-04$ \\
$(29)\left(\tau_{0}=0.25\right)$ & $1.32 \mathrm{e}-03$ & $4.13 \mathrm{e}-03$ & $3.37 \mathrm{e}-04$ & $1.03 \mathrm{e}-03$ & $8.44 \mathrm{e}-05$ & $2.59 \mathrm{e}-04$ \\
$(30)\left(\tau_{0}=0.5\right)$ & $1.34 \mathrm{e}-03$ & $4.31 \mathrm{e}-03$ & $3.37 \mathrm{e}-04$ & $1.07 \mathrm{e}-03$ & $8.47 \mathrm{e}-05$ & $2.69 \mathrm{e}-04$ \\
$(30)\left(\tau_{0}=0.25\right)$ & $1.33 \mathrm{e}-03$ & $4.26 \mathrm{e}-03$ & $3.36 \mathrm{e}-04$ & $1.06 \mathrm{e}-03$ & $8.43 \mathrm{e}-05$ & $2.66 \mathrm{e}-04$ \\
$(31)\left(\tau_{0}=0.5\right)$ & $1.67 \mathrm{e}-03$ & $3.44 \mathrm{e}-03$ & $4.22 \mathrm{e}-04$ & $8.70 \mathrm{e}-04$ & $1.06 \mathrm{e}-04$ & $2.18 \mathrm{e}-04$ \\
$(31)\left(\tau_{0}=0.25\right)$ & $1.67 \mathrm{e}-03$ & $3.45 \mathrm{e}-03$ & $4.22 \mathrm{e}-04$ & $8.71 \mathrm{e}-04$ & $1.06 \mathrm{e}-04$ & $2.18 \mathrm{e}-04$ \\
\hline
\end{tabular}

Table 4. The maximal error for $u_{x}(t, x, y)$ with the same parameters as in Table 3

\begin{tabular}{lcccccc}
\hline$\widetilde{h}$ & \multicolumn{2}{c}{$1 / 5$} & \multicolumn{2}{c}{$1 / 10$} & \multicolumn{2}{c}{$1 / 20$} \\
\hline$t$-interval & {$[0,0.5]$} & {$[0,1]$} & {$[0,0.5]$} & {$[0,1]$} & {$[0,0.5]$} & {$[0,1]$} \\
\hline$(27)$ & $2.42 \mathrm{e}-03$ & $8.52 \mathrm{e}-03$ & $6.16 \mathrm{e}-04$ & $2.16 \mathrm{e}-03$ & $1.54 \mathrm{e}-04$ & $5.39 \mathrm{e}-04$ \\
$(28)$ & $2.91 \mathrm{e}-03$ & $1.08 \mathrm{e}-02$ & $7.44 \mathrm{e}-04$ & $2.75 \mathrm{e}-03$ & $1.89 \mathrm{e}-04$ & $6.98 \mathrm{e}-04$ \\
$(29)\left(\tau_{0}=0.5\right)$ & $2.40 \mathrm{e}-03$ & $8.10 \mathrm{e}-03$ & $6.05 \mathrm{e}-04$ & $2.03 \mathrm{e}-03$ & $1.52 \mathrm{e}-04$ & $5.09 \mathrm{e}-04$ \\
$(29)\left(\tau_{0}=0.25\right)$ & $2.35 \mathrm{e}-03$ & $8.44 \mathrm{e}-03$ & $5.92 \mathrm{e}-04$ & $2.10 \mathrm{e}-03$ & $1.49 \mathrm{e}-04$ & $5.28 \mathrm{e}-04$ \\
\hline
\end{tabular}

\section{References}

[1] P. Besala, Finite difference approximation to the Cauchy problem for nonlinear parabolic differential equations, Ann. Polon. Math. 46 (1985), 19-26.

[2] D. Jaruszewska-Walczak, Difference methods for infinite systems of hyperbolic functional differential equations on the Haar pyramid, Opuscula Math. 24 (2004), 85-96.

[3] Z. Kamont, Hyperbolic Functional Differental Inequalities and Applications, Kluwer, Dordrecht, 1999.

[4] Z. Kamont and H. Leszczyński, Stability of difference equations generated by parabolic differential-functional problems, Rend. Mat. Appl. 16 (1996), 265-287.

[5] D. Kleitman, On a lemma of Littlewood and Offord on the distribution of certain sums, Math. Z. 90 (1965), 251-259.

[6] H. Leszczyński, Finite difference schemes for a non-linear heat equation with functional dependence, Z. Angew. Math. Mech. 79 (1999), 53-64.

[7] M. Malec, Sur une méthode des différences finies pour une équation non linéaire intégro-différentielle à argument retardé, Bull. Acad. Polon. Sci. Sér. Sci. Math. Astronom. Phys. 26 (1978), 501-512. 
[8] M. Malec, C. Mączka and W. Voigt, Weak difference-functional inequalities and their application to the difference analogue of nonlinear parabolic differential-functional equations, Beiträge Numer. Math. 11 (1983), 69-79.

Department of Differential Equations

Faculty of Applied Physics and Mathematics

Gdańsk University of Technology

Narutowicza 11/12

80-952 Gdańsk, Poland

E-mail: apoli@mif.pg.gda.pl

Received 2.8.2004

and in final form 5.1.2006 